\title{
Optimal itinerary planning for mobile multiple agents in WSN
}

\author{
Mostefa BENDJIMA ${ }^{1}$ \\ STIC Laboratory \\ University of Tlemcen \\ Tlemcen, Algeria
}

\author{
Mohamed FEHAM ${ }^{2}$ \\ STIC Laboratory \\ University of Tlemcen \\ Tlemcen, Algeria
}

\begin{abstract}
Multi mobile agents based on data collection and aggregations have proved their effective in wireless sensor networks (WSN). However, the way in which these agents are deployed must be intelligently planned and should be adapted to the characteristics of wireless sensor networks. While most existing studies are based on the algorithms of itinerary planning for multiple agents i.e. determining the number of mobile agents (MAs) to be deployed, how to group source nodes for each MA, attributing the itinerary of each MA for its source nodes. These algorithms determine the efficiency or effectiveness of aggregation. This paper aims at presenting the drawbacks of these approaches in large-scale network, and proposes a method for grouping network nodes to determine the optimal itinerary based on multi agents using a minimum amount of energy with efficiency of aggregation in a minimum time. Our approach is based on the principle of visiting central location (VCL). The simulation experiments show that our approach is more efficient than other approaches in terms of the amount of energy consumed in relation with the efficiency of aggregation in a minimum time.
\end{abstract}

Keywords-Wireless sensor networ; mobile agent; optimal itinerary; energy efficiency.

\section{INTRODUCTION}

A wireless sensor network (WSN) consists of a set of nodes capable of collecting information from a monitored environment and for transmission to a base station (Sink) via the wireless medium. WSNs are often characterized by dense deployment in large-scale environments that are limited in terms of resources. The limitations are the insufficient processing, storage capacities and especially energy shortage because they are usually powered by batteries. Constraint on the size of a sensor node requires designers to reduce the size of the battery and therefore the amount of energy available. Replacing a battery is rarely possible, for reasons of cost or constraints due to the environment. This leads to problems related to energy consumption during the operation of different network nodes.

Therefore, unlike traditional networks concerned with ensuring a good quality of service, WSNs must give equal importance to energy conservation. It is widely recognized that energy limitation is an unavoidable issue in the design of WSNs because it imposes strict constraints on the operation of the network. In fact, energy consumption of sensor nodes plays an important role in the life of the network, and has become the predominant criterion of performance in this area.
If we want the network to function satisfactorily as long as possible, these energy constraints force us to compromise between different activities, both at the node and network levels.

Several research studies have emerged with a goal: to optimize energy consumption of nodes through the use of innovative conservation techniques to improve network performance, including the maximization of its life. In general, energy economy remains the best compromise between the different energy-consuming activities. WSNs literature recognizes that the data is a prominent consumer of energy; due to this, the majority of work envisaged techniques affecting this section.

One technique for minimizing energy consumption in sensor networks, which are proposed by the authors such as [1] [2] [3] is the Mobile Agents (MAs) technique. In these proposals, it is crucial to find an optimal itinerary for a mobile agent to perform data collection from multiple distributed sensors.

Therefore, the MA is defined as a message that contains application code, a list of source node predefined by a Sink, and an empty field to put the data [4]. Among the disadvantages of this type of solution is the difficulty of creating areas that will be addressed by the MA. However, as a solution to optimize the itinerary of the MA in data fusion, [5] proposed two heuristic algorithms. A randomly selected itinerary may even cause worse performance than the traditional Client/Server model. For the Local Closest First (LCF) algorithm, the MA itinerary starts from a node and searches for the next destination with the shortest distance to its location. Concerning the Global Closest First (GCF) algorithm, the MA starts its itinerary from a node and selects the next destination closest to the center of the surveillance zone.

In addition, using only a single MA for sensor networks on a large scale can have the following drawbacks:

- A great delay with a large number of source nodes to visit;

- Sensor nodes in the itinerary of the MA deplete energy faster than other nodes;

- During the visit to source nodes, the size increases continuously MA; 
- MA transmission will consume more energy in its itinerary back to the sink;

- The increasing amount of data accumulated by the MA during its migration increases its chances of being lost due to the noise in the wireless medium;

- Thus, the longer the itinerary, the more risky is the agent-based migration.

To overcome this problem, several agents can be used, which gives rise to a new problem; itinerary planning for multi agents, which has a significant impact on the performance of the sensor network, knowing that it is crucial to find an optimal itinerary for each MA to visit all indicated source nodes.

Researchers have proposed to partition the network into groups such as cluster algorithms or visit central location with a special node as a Cluster-Head or center for each group respectively. This supports the data exchange with the base station, receiving sensored data from all nodes in one group to send the Sink.

In this paper, we present an approach adopted to solve the problem of itinerary planning for multiple agents to visit source nodes in each group in parallel. Iteratively, our algorithm partitions the network into sectors where source nodes in each sector are included in an itinerary. Source nodes in each sector can be obtained by choosing the angle in an adaptive manner.

The rest of the paper is organized as follows: Section 2 Provides an overview of the literature in which algorithms for a number of studies have been conducted for itinerary planning of mobile agents in the WSN, first we present the solutions by using a single mobile agent, then the solutions proposed for the use of multi mobile agents. Next, in section 3, we present the problem statement. Our proposed method for partitioning the network into sectors, with the Sink as a starting point, to solve the problem of itinerary planning for multiple agents to visit source nodes in each sector in parallel, is described in section 4. Then, Section 5 sets forth the purpose of our application, which is to establish a system to simulate the communication between a set of sensors and a base station forming a wireless sensor network. Finally, Section 6 summarizes and concludes this paper.

\section{StATE Of THE ART}

A number of studies have been conducted for mobile agent itinerary planning in the sensor network. For itinerary planning, first we present the solutions proposed by the use of a single mobile agent, then we focus on the solutions proposed for the use of multi mobile agents.

\section{A. The use mobile agent}

Itinerary planning of a single mobile agent has a significant impact on the effectiveness of sensor networks. It determines the order of visits to source nodes during mobile agent migration.Among these proposals, Local Closest First (LCF) and Global Closest First (GCF) are simple approaches to itinerary planning of a single mobile agent [6]. The LCF algorithm seeks the source node with the shortest distance from the current node, while the GCF algorithm searches for the following source node, which has the shortest distance from the Sink. The author Chen proposed Mobile-AgentDirected Diffusion (MADD) algorithm in [7] that is similar to the LCF, but chooses the farthest source node as the starting point of the itinerary.

There are LCF algorithms that extend by estimating the cost of communication in the migration of the MA, with the increase of the MA packet size. Among these algorithms [8], Itinerary Energy Minimum for First source selection (IEMF) and Itinerary Energy Minimum Algorithm (IEMA) have the highest performance in terms of energy efficiency and delay compared to existing solutions. To address this performance, a simplified analytical model is used to formulate the itinerary calculated by an objective function directly proportional to the received signal strength and inversely proportional to data loss and energy consumption.

Restrictions on the use of a single agent to perform the entire task make the algorithm unreliable in applications where a large number of sources nodes are to be visited.

Typically, itinerary algorithms with a single agent have a high efficiency when the source nodes are distributed geographically close to each other, and the number of source nodes is not high.

For sensor networks on a large scale, in which many sources nodes have to be visited, the itinerary of a single mobile agent presents the following disadvantages:

- Significant delays when a single agent has visited hundreds of sensor nodes;

- Sensor nodes in the itinerary of the mobile agent deplete energy faster than other nodes;

- The size of the mobile agent increases when visiting more source nodes, so during transmission, the agent will consume more energy in its itinerary back to the base station;

- Reliability is reduced when the agent accumulates an increasing amount of data;

- When the mobile agent migrates to several source nodes, the chance of being lost increases.

To address these problems, multi mobile agents can be used, which causes a new problem requiring itinerary planning for multiple agents.

\section{B. The use of multiple mobile agents}

Chen et al. [9] proposed the algorithm Centre Locationbased Multi agents Itinerary Planning (CL-MIP), the main idea is to consider the solution of multi agent itinerary planning (MIP) as an iterative version of the solution the single mobile agent itinerary planning (SIP).

Contributions of the CL-MIP include the following:

- It first proposes a generic framework in several steps to solve the MIP problem, which reuses existing solutions in SIP;

- The proposed gravity algorithm accurately describes the density center of source node groups, which is the basis of the source group. 
Another algorithm, Genetic Algorithm-based Multi agents Itinerary Planning (GA-MIP) is proposed in [10]. GA-MIP first proposes a new method for two-level encoding of MA to solve the MIP problem. The GA-MIP algorithm considers the problem as a single problem instead of using several steps, as adopted by the previous algorithms.

The proposal in [11] considers models of MIP problems as a Totally Connected Graph (TCG). In the TCG, the vertices are the nodes of the sensor network, and the weight of an edge is derived from estimates of the jump between the two end nodes of the edge. The authors indicate that all source nodes in a particular sub-tree should be considered as a group. In addition, the authors present a balancing factor while calculating the weight in the TCG, to form a minimum Balanced Spanning Tree (BST). The balancing factor allows flexible control over the compromise between energy cost and duration of the task. This algorithm is named Balanced minimum Spanning Tree-based Multi agents Itinerary Planning (BST-MIP). The main contribution of BST-MIP is that it analyzes the critical impact of geographical positions on the source node group.

Mpitziopoulos et al. in [12] proposed a Near-Optimal Itinerary Design (NOID) algorithm to solve the problem of computing a near-optimal itinerary for MA. NOID algorithm was designed so as to adapt rapidly to changing conditions of the network, the MA itinerary must contain only source nodes with enough energy availability and the number of AMs should depend on the number of physical locations of the source nodes to be visited.

Gavalas et al. in [13] presented the second quasi-optimal itinerary design algorithm (SNOID) to determine the number of mobile agents that should be used, and the itinerary of these mobile agents should follow. The main idea behind SNOID is to partition the area around the Sink into concentric zones and begin to build paths for MA with the direction of the inside near Sink. The radius of the first area that includes Sink equals (a.rmax) where a is an input parameter in the range $[0,1]$ and rmax is the maximum transmission range of any source node. All source nodes inside the first zone are connected directly to Sink, and are the starting points of mobile agent itinerary.

\section{PROBlem STATEMENT}

\section{A. VCL algorithm}

The author of [1] proposed selection algorithm of the Visiting Centre Local (VCL), it assumes that we have sensor nodes $\mathrm{n}$, so this algorithm is based on the principle of distributing the impact factor of each sensor node to other sensor nodes. Then, each sensor node will receive (n-1) impact factors from other sensor nodes, and of itself. After calculating the accumulated impact factor, the location of the sensor node with the largest cumulative impact factor is selected as (VCL).We denote the set of $\mathrm{n}$ nodes in $\mathrm{V}$.

For two source nodes $\mathrm{i}, \mathrm{j} \in \mathrm{V}, \mathrm{d}(\mathrm{i}, \mathrm{j})$ is the distance between $i$ and $j$. Then, we can estimate the number of hops between these two source nodes as: $H_{i}^{j}=\frac{d(i, j)}{R}$ where $R$ represents the maximum transmission range. To approximate the effects of a real gravitational field, a Gaussian function is adopted to calculate the impact factor between two source nodes $\mathrm{i}$ and $\mathrm{j}$ : $\mathrm{G}_{\mathrm{ij}}=\mathrm{e}^{-\frac{\left.\left(\mathrm{H}_{\mathrm{i}}^{\mathrm{j}}-1\right)\right)^{2}}{2 \sigma^{2}}}$, where $\sigma$ is a constant, set to 08 in all previous works.

\section{B. LCF algorithm}

We chose the algorithm Local Closest First (LCF) to trace the itinerary of the MA as it allows finding an optimal way to avoid redundancies, and to manipulate lists of nodes in a consistent manner. According to [5] the itinerary starts from the local point of a sub-area, our strategy aims requires the Sink to be the starting points for the packet itinerary.

\section{DESCRIPTION OF THE APPROACH}

The main purpose of our strategy is to partition the nodes of wireless sensor network into groups. The partition is done iteratively so that source nodes are included in an itinerary. The data, collected by the source nodes in each group, are arranged in a group. Then, the data of each group of source nodes are sent as a pack instead sending them separately by each source nodes to the Sink.

The main idea is to partition the network into sectors, with the starting point is the base station. For each sector, we propose a mobile agent to collect information from these source nodes along an itinerary similar in shape to the sector.

Once the Sink receives signals from source nodes, it will send mobile agents, so that they circulate between its nodes using the itinerary Local Closest First algorithm (LCF). The mobile agents aggregate data processed and collected by the nodes to return to the Sink with the information collected.

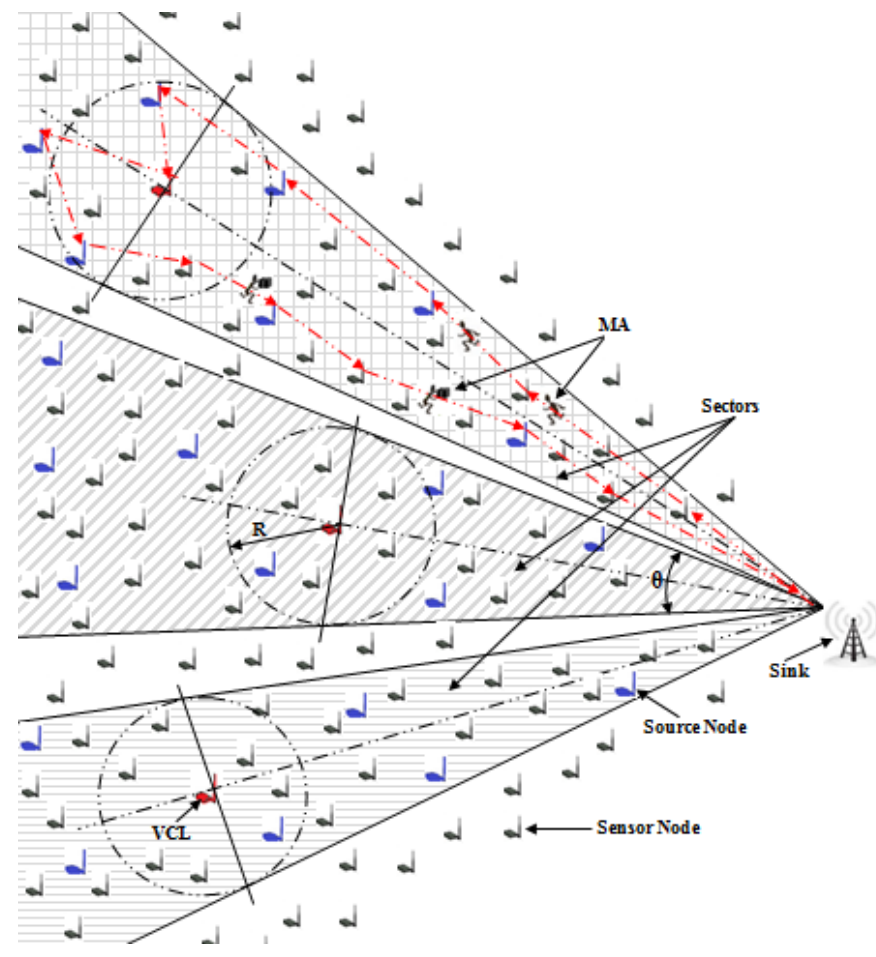

Figure 1. Source nodes partitioning into sectors 
As shown in "Fig.1", the disk radius is an important parameter in source nodes grouping. Similarly, the selection of the angle $\theta$ determines the size of the sector, which is important for an efficient itinerary. Data aggregation of MAs by the source nodes from all sectors passes through the following steps:

- calculating the VCL source nodes;

- Constructing an angle sector $\theta$ and a right-side center between the Sink and VCL;

- Grouping the source nodes inside the sector;

- We repeat these steps for all the remaining source nodes;

After that, all source nodes are sources included into the sectors, a mobile agent is assigned to each sector. At this point, the itinerary of the MA can be planned for each sector, using the LCF algorithm.

All these steps are translated in three algorithms using the following notation:

NOTATION:

VCL: visiting center local;

$\theta$ : angle of the sector;

$\mathrm{R}$ : maximum transmission range;

$\mathrm{S}$ : sector for source node grouping;

$\mathrm{T}$ : set of source nodes to be grouped in $\mathrm{S}$ sectorateachiteration;

$\mathrm{N}$ : total number of nodes of sources;

E: set of source nodes;

E': set of remaining source nodes;

$\mathrm{SN}$ : source node.

\section{ALGORITHM 01: ALGORITHM FOR PARTITION INTO SECTORS BEGIN}

- $\quad$ Plotting a straight line connecting the Sink and the VCL;

- Plotting a straight line perpendicular to the line connecting the Sink and the VCL (Sink, VCL), the $V C L$ is the point of intersection;

- $\quad$ Plotting a circle (disk) whose center is the VCL and with a radius $R$;

- Connecting the two points of intersection of the straight line perpendicular and the circle with the Sink;

End.

Algorithm 02: algorithm to group nodes of sources

Begin

$T=\{\}$;

For each source node SN in E do

If $S N \in S$ then
$\mathrm{T}=\mathrm{T} \cup\{\mathrm{SN}\}$

$\mathrm{E}=\mathrm{E}-\mathrm{SN}$

END IF;

END FOR;

END.

ALGORITHM 03: ALGORITHM FOR ITINERARY PLANNING MMA (Multi MOBILE AGENT)

Begin

While $\mathrm{E}<>\{\}$ do

Selection algorithm VCL / / (see VCL algorithm [14]);

Partitioning algorithm into sectors / / (see Algorithm 01);

Algorithm to group source nodes / / (see Algorithm 02);

$\mathrm{E}=\mathrm{E}-\mathrm{T}$;

End while;

Determine the itinerary of AM in each sector area by LCF (see LCF algorithm [05]);

End.

\section{Simulation SETUP}

\section{A. Purpose of the software}

For our simulation platform, we have established a system to simulate communication between a set of sensors and a base station constituting a wireless sensor network. The technique we used consists in partitioning the network into sectors, and using mobile agents, in order to save the network energy. So the goal of our application is to carry out itinerary planning of mobile multi agents that is effective in terms of energy and in terms of task duration.

$B$. Initial hypotheses

To perform the simulation, we adopted the following assumptions:

- At the beginning, each sensor has an initial energy;

- We can choose the number of nodes, the radius and position of Sink;

- The number of sensors is limited by a minimum and a maximum value;

- The positions of the sensor nodes are known to the Sink;

- We can add sensor nodes before the activation of the nodes only;

- The deployment is random;

- Two sensors do not occupy the same coordinates;

- We consider that the problem of failure is caused by a depletion of energy;

- The simulation stops when a target cannot be covered by at least one sensor;

- The aggregation model and energy used is mentioned in reference [15]. 
We performed our simulations on a $1000 \mathrm{~m} \times 500 \mathrm{~m}$ with a random distribution of 1000 sensor nodes for 1000 seconds. Thus, the base station is located to the right of the field, and multiple source nodes are randomly distributed in the network. We have limited the radio coverage, and brought the data rate of each node to 80 meters and $1 \mathrm{Mbps}$, respectively, as suggested in [16]. The parameters of power transmission and reception, which directly affect the radio range, were selected from the ranges defined in the sunspot system [17]. Local processing time is $40 \mathrm{~ms}$. The parameters used for our simulation are summarized in Table I.The values we used are inspired by the work done by [18].

TABLE I: BASIC SIMULATION PARAMETERS

\begin{tabular}{|l|l|}
\hline Simulation parameter & Values \\
\hline Node distribution & Random \\
\hline Radio range & $80 \mathrm{~m}$ \\
\hline Debit & $1 \mathrm{Mbps}$ \\
\hline Data collected interval & 10 seconds \\
\hline Simulation time & 1000 seconds \\
\hline Local processing time & $40 \mathrm{~ms}$ \\
\hline Processing code size & $0.4 \mathrm{Ko}$ \\
\hline Raw Data Size & 2024 bits \\
\hline MA Code Size & 1024 bits \\
\hline MA Accessing Delay & $10 \mathrm{~ms}$ \\
\hline Data processing Rate & $50 \mathrm{Mbps}$ \\
\hline Raw Data Reduction Ratio & 0.8 \\
\hline Aggregation Ratio & 0.9 \\
\hline Network Size & $1000 \mathrm{~m} \times 500 \mathrm{~m}$ \\
\hline Number of Sensor Nodes & 1000 \\
\hline
\end{tabular}

\section{Results and analysis}

Itinerary planning for a single mobile agent allows determining the order of source nodes visited during migration of a mobile agent, it has a significant impact on the effectiveness of sensor networks MA-based. Several studies have been conducted for itinerary planning in a single mobile agent in a WSN. Among these works, Local Closest First (LCF) and Global Closest First (GCF) are simple approaches [19] for itinerary planning of a single mobile agent. LCF seeks the source node according to the shortest distance from the current node, while the GCF seeks the source node which has the shortest distance from the base station.

To demonstrate the performance of our Multi Mobile Agent Itinerary (MMAI) approach in wireless sensor networks, we compare it with LCF and GCF. In this section, we examine the impact of the number of source nodes on the energy performance and the duration of tasks. Sensor nodes are energy limited, except for the Sink which is assumed to have infinite energy input. We assume that the Sink and sensor nodes are stationary and that the Sink is located on the right side of the field. To check the scaling property of our algorithms, we select a large-scale network with 1000 nodes, so we set the number of source nodes from 05 to 50 by steps of 05 , and get a set of results for each case. We represented the results obtained in "Fig. 2", which shows the impact of the number of nodes on energy sources, for sensory data of all source

nodes.

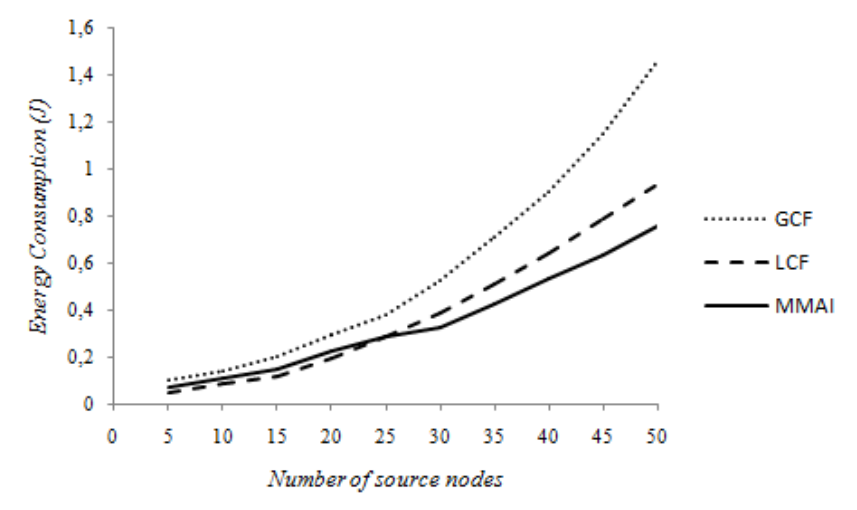

Figure 2. Energy consumption by the number of source nodes

The analysis of the "Fig. 2" highlights several interesting elements: first, which is quite normal, when the number of source nodes is increased, more energy is required for mobile agents to perform tasks for each of the three approaches. However, for an equal number of source nodes, consumption using our MMAI approach is always lower than that of LCF and GCF. Moreover, the difference between our approach and the approach GCF starts digging from 05 source nodes, while the gap with other LCF begins at 25 sources nodes. When the number of nodes is low, energy consumption of our approach is less important compared to LCF, between 05 and just under 25 nodes, the latter achieves an additional energy saving $18 \%$ higher than MMAI. It may be noted again that from 25 source nodes the difference between MMAI and the other two approaches is becoming increasingly important, and this difference increases continuously with increasing number of nodes. Nevertheless, from just over 25 nodes, our MMAI approach significantly outperforms the two other approaches; for example, at 30 nodes; LCF and GCF consume $16 \%$ of energy and $37 \%$ more than MMAI, respectively. However, at 50 source nodes, consumption using MMAI approach is $21 \%$ lower than in the LCF approach and $48 \%$ less compared to the GCF approach. By comparison, the multi mobile agent itinerary solution has better energy efficiency.

In addition, and in another experiment, we show the performance comparison of the three approaches in terms of task duration. The results are shown in "Fig. 3".

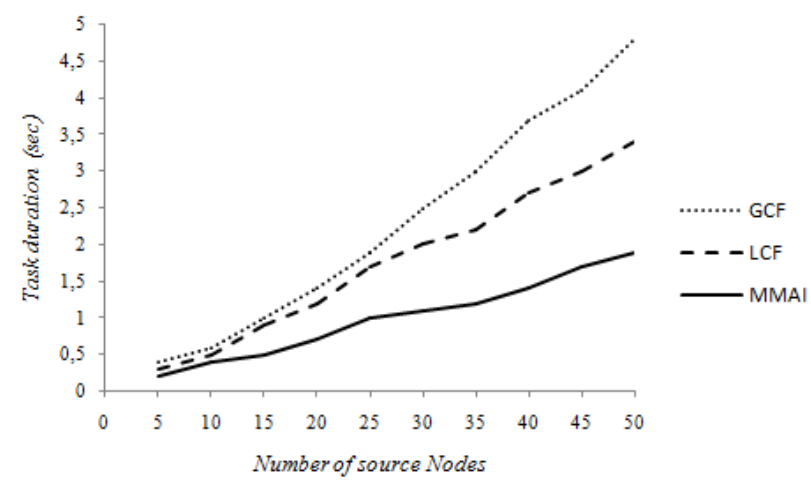

Figure 3. Task duration made by the number of nodes 
"Fig. 3" compares the performance of the three approaches in terms of the duration of tasks. We note that in terms of task duration our approach is still shorter than that of other approaches, regardless of the number of source nodes. In the single mobile agent approach as LCF and GCF, the duration of a task starts from the moment the mobile agent is distributed by the base station until the agent returns to the station.

In multi mobile agent approach such as MMAI, since many mobile agents are working in parallel, there must be an MA which is the last to return to the Sink. Then, the duration of the task of the MMAI approach is the delay of that agent.

Compared to the energy performance, the number of nodes sources has a greater impact on the delay of the task. First, when the number of source nodes is increased, a longer period of time is required for mobile agents to perform the duties of each of the three approaches. It may be noted again that the difference between our proposal and the other two approaches is becoming increasingly important, and this difference increases continuously with the increasing number of source nodes. The duration of a task in an approach with a single mobile agent as LCF and GCF becomes much greater for a greater number of source nodes, because with more source nodes to visit, the size of an MA becomes bigger, and more transmissions are made.

At the beginning, the three approaches are almost similar, but at 25 source nodes, LCF and GCF consume $41 \%$ and $48 \%$ more time than MMAI, respectively. Our approach is advantageous with increasing source nodes, nodes with 50 sources, MMAI minimize delay, over $44 \%$ in LCF, and approximately $61 \%$ in GCF.

The reason for this result is that in the itinerary of a single MA, only one MA moves along the entire network to collect information from all source nodes. This procedure leads to a greater latency from source nodes that can be distributed throughout the network. A multi agents approach can speed up the task because several itineraries are applied simultaneously. As Figure 03 shows, the duration of the task of the proposed MMAI is still the lowest in comparison with the other two approaches.

\section{CONCLUSION AND PeRsPectives}

In this paper, we proposed a solution to the problem of itinerary planning for multiple in wireless sensors networks. Recent research has demonstrated the effectiveness of using a single mobile agent for data collection in wireless sensor networks. Using a single mobile agent may be deficient, for example, the increased latency in task completion and unbalanced energy consumption by the nodes. Nevertheless, it is crucial to find an optimal itinerary for each MA to visit all the source nodes. To remedy this problem, we partitioned the network into sectors, and within each sector, we use a mobile agent to collect information from these source nodes along an itinerary with a shape similar to the sector. Therefore, we used multi mobile agents to visit the source nodes in each group in parallel, which facilitates the simultaneous collection of sensory data to reduce the duration of tasks in depth. Iteratively, our algorithm partitions the network into areas where the nodes in each sector sources are included in an itinerary; the starting point is the base station. Once the Sink receives signals from source nodes, it will send mobile agents, so that they circulate between its nodes using the algorithm groups LCF. These mobile agents perform data aggregation of processed and collected data by the source nodes to return to Sink with the information collected.

Many simulations have been performed to show the superior performance of our MMAI approach compared to LCF and GCF approaches in terms of the duration of tasks and energy consumption. It saves significant energy and thus significantly reduces costs on a large scale.

For future work, we will attempt to improve the selection method of the angles of the sector, so that the optimal itinerary for MAs can be obtained by selecting the effective angle in an adaptive mode.

\section{REFERENCES}

[1] M. Chen, T. Kwon, Y. Yuan, and V. C. M. Leung, "Mobile Agent Based Wireless Sensor Networks," pp. 14-21, 2006.

[2] T. Lang, Z. Qing, and A. Srihari, "Sensor networks with mobile agents," Multidisciplinary University Reseanh Initiative (MURI) under the Omce of Naval Research and Army Rerearch Laboratory CTA on Communication and Network underGrant DAADIP-OI-2CQII, 2003.

[3] Q. Wang, and H. Xu, "Mobile-agent-based collaborative signal and information processing in sensor networks," Proceedings of the IEEE 91(8), 2003.

[4] M. Ketel, S. Numan, and A. H. Dogan, "Distributed Sensor Networks based on Mobile Agents Paradigm," Dept. of Electrical \& Computer Eng.North Carolina A\&T State University Greensboro, NC 27411. IEEE, 2005.

[5] Q. Hairong, and W. Feiyi, "Optimal Itinerary Analysis for Mobile Agents in Ad Hoc Wireless Sensor Networks," University of Tennessee, Knoxville, Advanced Networking Group MCNC, Research Triangle Park, 2001.

[6] F. Mourchid, "Nouveau Modèle pour le Positionnement des Senseurs avec Contraintes de Localisation," memoire the master applied sciences, AVRIL 2010.

[7] M. Chen, "Mobile Agent-Based Directed Diffusion in Wireless Sensor Networks," EURASIP J. Advances in Processing, vol. 2007.

[8] M. Chen, T. Laurence, T. Yang, and Z. Liang, "Itinerary Planning for Energy-Efficient Agent Communications in Wireless Sensor Networks," IEEE TRANSACTIONS ON VEHICULAR TECHNOLOGY, VOL. 60, NO. 7, SEPTEMBER 2011.

[9] M. Chen, S. Gonzlez, Y. Zhang, and V.C. Leung, "Multi-agent itinerary planning for sensor networks," Proc. IEEE 2009 Int. Conf. Heterogeneous Networking for Quality, Reliability, Security and Robustness (QShine 2009), Las Palmas de Gran Canaria, Spain,2009.

[10] W. Cai, M. Chen, T. Hara, and L. Shu, "GA-MIP: genetic algorithm based multiple mobile agents itinerary planning in wireless sensor network," Proc. Fifth Int. Wireless Internet Conf. (WICON), Singapore, 2010.

[11] M. Chen, W. Cai, S. Gonzalez, and V.C. Leung, "Balanced itinerary planning for multiple mobile agents in wireless sensor networks. Proc. Second Int. Conf. Ad Hoc Networks (ADHOCNETS2010), Victoria, BC, Canada, 2010.

[12] D. Gavalas, A. Mpitziopoulos, G. Pantziou, and C. Konstantopoulos, "An approach for near-optimal distributed data fusion in wireless sensor networks," Springer Wirel. 16, (5), pp. 1407-1425, 2009.

[13] P Sameera, P.Sundeep, K. Bhaskar, and S. Gaurav, "A unifying framework for tunable topology control in sensor," University of Southern alifornia, USA, 2005.

[14] M. Chen, V. Leung, S. Mao, and T. Kwon, "Energy-efficient Itinerary Planning for Mobile Agents in Wireless Sensor Networks," In: IEEE. 2007. International Conference on Communications, Dresden, Germany, 2009. 
[15] C. Konstantopoulos, A. Mpitziopoulos, D. Gavalas, and G. Pantziou, "Effective determination of mobile agent itineraries for data aggregation on sensor networks," IEEE Trans. Knowl. Data Eng. pp. 1679-1693, 2010.

[16] K. Sohraby, and D. Minoli, "Wireless Sensor Networks, Technology, Protocols, and Applications," WILLEY, 2007.

[17] SunTM, Small Programmable Object Technology (SunSPOT) Theory of Operation. Technical report, Sun Microsystem, SunLabs, 2008.
[18] A.sardouk, and 1. boulahia, "agent-cooperation based communication architecture for wireless sensor network," in: ifip/ieee wireless days/adhoc and wireless sensor networks, uae, ifip, 2008.

[19] M. chen, s. gonzlez, y. zhang, and v. leung, "multiagent itinerary planning for sensor networks," in proceedings of the iee 2009 international conference on heterogeneous networking for quality, reliability, security and robustness (qshine 2009), laspalmas de gran canaria, spain, 2009. 